\title{
Germanica
}

\section{Einmal Leningrad - Wien - New York und zurück. Stationen einer Odyssee in Vladimir Vertlibs Roman Zwischenstationen}

Leningrad - Wien - New York und zurück. Étapes d'une odyssée dans le roman de Vladimir Vertlib Zwischenstationen.

\section{Alfred Strasser}

\section{OpenEdition Journals}

Édition électronique

URL : http://journals.openedition.org/germanica/378

DOI : 10.4000/germanica.378

ISSN : 2107-0784

\section{Éditeur}

Université de Lille

\section{Édition imprimée}

Date de publication : 1 juin 2006

Pagination : 103-113

ISBN : 2-913857-17-5

ISSN : 0984-2632

\section{Référence électronique}

Alfred Strasser, « Einmal Leningrad - Wien - New York und zurück. Stationen einer Odyssee in Vladimir Vertlibs Roman Zwischenstationen », Germanica [Online], 38 | 2006, Online erschienen am: 19 Februar 2010, abgerufen am 06 Oktober 2020. URL : http://journals.openedition.org/germanica/378 DOI : https://doi.org/10.4000/germanica.378

Ce document a été généré automatiquement le 6 octobre 2020.

(c) Tous droits réservés 


\title{
Einmal Leningrad - Wien - New York und zurück. Stationen einer Odyssee in Vladimir Vertlibs Roman Zwischenstationen
}

\author{
Leningrad - Wien - New York und zurück. Étapes d'une odyssée dans le roman \\ de Vladimir Vertlib Zwischenstationen.
}

Alfred Strasser

„Es ist schon tragisch“, sagte Rita, nachdem sie sich wieder gesetzt hat, „Russen durftet ihr nicht

sein, richtige Juden seid ihr keine mehr, Gojim aber auch nicht."

\section{I}

1 Einer der produktivsten zeitgenössischen Autoren Österreichs ist zweifellos der aus der Sowjetunion stammende Vladimir Vertlib. Seine erste Novelle Das Bett erschien 1993 in der Zeitschrift der Theodor-Kramer-Gesellschaft ${ }^{2}$ „Mit der Ziehharmonika“, seitdem hat er vier Romane und einige Erzählungen veröffentlicht. Dem ersten Roman Abschiebung (1995) folgte 1999 Zwischenstationen, danach legte er 2001 Das besondere Gedächtnis der Rosa Masur vor und schließlich erschien zwei Jahre später sein bis jetzt letzter Roman Letzter Wunsch (2003); sein neuestes Buch ist ein Erzählungenband und heißt Mein erster Mörder (2006). Vertlib schreibt außerdem regelmäßig für verschiedene Periodika, insbesondere für die Zeitschrift „Zwischenwelt“, der Nachfolgepublikation von,Mit der Ziehharmonika“,die sich vor allem damit beschäftigt, das Leben der österreichischen Exilanten während der NS-Zeit zu erforschen.

2 Exil und Emigration ist auch das Stichwort, das am besten die zwanzig ersten Lebensjahre Vladimir Vertlibs charakterisieren. Geboren 1966 im damaligen Leningrad, 
emigrierte er im Alter von fünf Jahren mit seinen Eltern nach Israel; das Leben in ihrer neuen Heimat entspricht aber nicht den Erwartungen der Eltern, sodaß sie das Land wieder verlassen und sich auf eine zehnjährige Odyssee begeben:

Die Emigration meiner Eltern, die 1971, als ich noch keine fünf Jahre als war, aus politischen Gründen, um dem staatlichen und alltäglichen Antisemitismus $\mathrm{zu}$ entfliehen, ihr Heimatland verlassen mußten, hatte mehrer Stationen: Israel Österreich - Italien - Österreich - Niederlande, wieder Israel - wieder Italien wieder Österreich - USA - und schließlich endgültig Österreich. Der Einwanderungsversuch in die USA endete mit Schubhaft und Abschiebung. Was ursprünglich nur als Übersiedlung aus der UdSSR nach Israel geplant war, entwickelte sich zu einer Anzahl von weiteren Emigrationen und Remigrationen, eine mehr als zehn Jahre dauernde Pendelroute zwischen verschiedenen Exilstationen. ${ }^{3}$

3 Schließlich wird ab 1981 Österreich Vertlibs ständige Bleibe. Er legte die Matura ab, studierte in Wien Volkswirtschaftslehre, und nach dem Abschluß seines Studiums arbeitete er zunächst als Statistiker bei einer Versicherung, später als Länderanalytiker für eine Bank. In dieser Zeit fing er an, gelegentlich zu schreiben, zuerst auf Russisch, manchmal auf Englisch, und schließ entschied er sich, seine Texte nur mehr auf Deutsch zu verfassen.

4 Vertlib ist einer jener sowjetischen Juden, die in den siebziger Jahren in großer Zahl die Sowjetunion verlassen haben, die meisten mit dem Ziel, in den Vereinigten Staaten oder in Israel eine neue Heimat zu finden. Österreich war für diesen Migrantenstrom eines der wichtigsten Transitländer; zwischen 1968 und 1986 reisten 270199 sowjetische Juden über Österreich in den Westen, und bis zu seiner Schließung ${ }^{4}$ war für viele Emigranten das Transitlager Schönau die erste Durchgangsstation auf ihrem Weg in die USA, die aber in den achtziger Jahren des vergangenen Jahrhunderts ihre Grenzen weitgehend schlossen, so daß ab diesem Zeitpunkt Israel das erste Ziel der Auswanderer wurde. Einige siedelten sich auch in Europa an, etwa in den skandinavischen Ländern oder in Italien, immerhin blieben zwischen 60000 und 100000 in Deutschland ${ }^{5}$ und einige, ganz wenige, auch in Österreich. ${ }^{6}$ Deutschland und Österreich waren dabei immer noch mit der Hypothek ihrer Nazivergangenheit belastet, die viele jüdische Auswanderer zögern ließ, sich dort niederzulassen, auch Vertlibs Eltern:

Daß meine Eltern in Österreich, der Heimat Hitlers und vieler kleinerer Nazis, keine Zweite Heimat sehen wollten, ist verständlich. Während des Krieges waren zahlreiche Angehörige ihrer Familien von den deutschen Besatzern in Weißrußland ermordet worden, und noch vor zwanzig Jahren hätte jeder dritte Passant, der durch die Straßen Wiens spazierte, unter Umständen einer dieser Mörder sein können. ${ }^{7}$

5 Nichtsdestotrotz ließ sich die Familie in Wien nieder, auch wenn dieser Schritt nach der Zwangsabschiebung aus den Vereinigten Staaten eher das Resultat von Behördenentscheidungen war als eine freiwillig getroffene Wahl: Des Wanderns müde, blieb die Familie schließlich in dem Land, das bereit war, ihr Aufenthaltspapiere zu geben. 

einer neuen Heimat, die mit der Suche nach der eigenen Identität verbunden ist, sind die Hauptthemen in Vertlibs autobiographischem Roman Zwischenstationen. Die im Roman beschriebenen Orte entsprechen der Odyssee Vertlibs von Leningrad bis Wien. Der Roman beginnt mit der Rückkehr des Ich-Erzählers in dessen alte Heimat, die jetzt nicht mehr Leningrad, sondern St. Petersburg heißt. Über zwanzig Jahre nach seiner Auswanderung besucht er die Großmutter, die kurz darauf sterben wird. Keine nostalgischen Gefühle bemächtigen sich seiner, als er in St. Peterburg aus dem Zug aussteigt; er hat von seinem ersten Lebensabschnitt Distanz genommen, denn sogar die topographischen Erinnerungen der frühen Kindheit sind brüchig geworden:

Unser Haus finde ich nicht gleich. Die weite Fläche zwischen der Nordbahnstraße und der alten Wohnsiedlung ist inzwischen verbaut worden. Ich muß den Stadtplan zu Hilfe nehmen. Schließlich stehe ich vor dem Tor. Das Haus wirkt abgewohnt. Im Flur riecht es nach Katzenpisse. (Z. 13)

Der Besuch in Rußland wird zur Rechtfertigung und zur Bestätigung der damaligen elterlichen Entscheidung, die Sowjetunion zu verlassen, deren Existenz 1971 für ihre Bewohner noch „für mindestens zweihundert Jahre fest[stand]“ (Z. 9). Zwar ist die Diktatur des Proletariats überwunden, doch die sie ersetzende neue Gesellschaftsform macht das Leben nicht einfacher und angenehmer. Im Gegensatz zu früher ist aber nun jeder für sein eigenes Glück oder Unglück verantwortlich: „Das Leben sei gefährlich und unberechenbar geworden [...] Am Abend auszugehen könne geradezu das Leben kosten. [...] Wer die Gefahren mißachte, sei selber schuld.“ (Z. 12) Wie wenig die vielen Verlierer mit der neuen Freiheit des „selbstverschuldeten“ Unglücks fertig werden, wird dem Erzähler in der Straßenbahn klar, als das Gespräch der klagenden Passanten, ganz selbstverständlich auf die ,jüdische Mafia“ kommt, die die Schuld an der Misere des Staates trage. Der in den neunziger Jahren wieder populär gewordene offene Antisemitismus ist ein zusätzliches Argument für die Richtigkeit der Entscheidung auszuwandern. Das hastige Hinunterschlingen einer Schokolade, die ihm zwar Übelkeit verursacht, gleich nachdem er Rußland wieder verlassen hat, wird zum symbolischen Akt der zwar schmerzhaften, aber endgültigen Loslösung.

Der Besuch des Ich-Erzählers in St. Petersburg ist ein Endpunkt, eine Bestätigung, daß er nach einer zehnjährigen Wanderschaft durch diverse Länder und Kontinente definitiv in seiner neuen Heimat Wien angekommen ist, aber gleichzeitig auch der Ausdruck, daß er definitiv eine neue Identität angenommen hat, nachdem sich lange, je nach dem momentanen Aufenthaltsort, verschiedene Identitäten übereinander stülpen ließen, eine russische, eine jüdische, eine amerikanische und eine österreichische und alle diese Identitäten miteinander vereinbar schienen. Sinnbild dafür ist ein Haus in Wien, das vorwiegend von ausgewanderten sowjetischen Jude als Durchgangsquartier benutzt wurde: „Ich dachte manchmal, ich sei in Israel, dann wieder, ich sei in Rußland, bis ich verstand, daß beides stimmte. Das Haus war ein Teil Israels und Rußlands, der sich in einem fremden Ort namens Wien befand. Keine Frage: die Welt war wie eine Anzahl von Schachteln aufgebaut, die ineinanderpaßten." (Z. 31) 
Ursprünglich wollte seine Familie, wie sehr viele sowjetische Juden, in Israel ihr neues Zuhause finden, doch schon die ersten Tage im Staat der Juden erweisen sich als desillusionierend; auch hier leben nicht alle „Menschen wie Brüder und Schwestern in Eintracht", nicht alle Bürger werden von den Behörden gleich behandelt, und die Einwanderer aus der Sowjetunion, die gewohnt waren, daß der omnipräsente Staat sich um alles kümmert, fühlen sich verraten, weil sie in Israel eigeninitiativ handeln müssen:

Man läßt die Leute kommen, und der Staat kümmert sich einen Dreck um seine neuen Bürger oder überantwortet sie der Willkür der Bürokratie. Jeder kämpft für sich allein. Alle gegen- und nicht füreinander. Wer Beziehungen oder Geld hat, der kann sich's richten. Wer Pech hat, landet in der Wüste. (Z. 38)

In den Bereichen, wo sich der Vater des Erzählers jedoch eine unterschiedliche Behandlung der Einwanderer durch die Behörden wünschen würde, geschieht dies nicht und alte Ressentiments aus der Zeit in der Sowjetunion werden wach: Die Familie lebt in ihrem Dorf in Israel nicht mit anderen intellektuellen Großstädtern aus dem europäischen Teil der Sowjetunion zusammen, sondern mit Emigranten aus dem verabscheuten Usbekistan, Tadschikistan und Kirgisien, Menschen, denen man in der Sowjetunion aus dem Weg gegangen wäre.

Zudem ist die israelische Gesellschaft genauso wenig frei von Rassismus wie die anderer Länder; er richtet sich zum einen gegen die „Schwarzen“, die aus Nordafrika stammenden, „schmutzigen, arbeitsscheuen und verbrecherischen“ Juden, vor denen die Neuankömmlinge sofort gewarnt werden, und gegen die Nichtjuden. Auch wenn sich die Bevölkerung des Landes vornehmlich aus ehemaligen Emigranten zusammensetzt, haben es in Israel Zuwanderer nicht leichter als in anderen Ländern, vor allem wenn sie, wie die Ehefrau eines ukrainischen Einwanderers, nicht jüdischen Glaubens sind:

In der Ukraine war mein Sohn mit seinem Aussehen und seinem Familiennamen eindeutig ein der Jud, in der Schule hätte man ihn zweifellos als Saujud bezeichnet [...] In Israel ist er hingegen $a$ Goi, weil seine Mutter Nichtjüdin ist. Wo, frage ich Sie [...] soll da die Gerechtigkeit sein. (Z. 28)

12 Die Integration der Familie des Erzählers wir vor allem aber dadurch erschwert und scheitert schließlich zwei Mal, weil sie keinerlei Bezug zum Judentum hat. Atheisten in der Sowjetunion, hat sich bei ihnen durch den dort herrschenden Antisemitismus zwar ein jüdisches, aber kein zionistisches Bewußtsein gebildet. Auch in Israel bleibt sie ihrer atheistischen Weltanschauung treu, sodaß Religion als möglicher Integrationsfaktor wegfällt und die zwei Versuche, sich in Israel niederzulassen, scheiterten: Beide Male verlassen sie das Land wieder kurz nach ihrer Einreise.

Trotz all offensichtlicher Schwächen bleibt Israel für die sowjetischen Emigranten der Ausnahmestaat, auch wenn sie dort nicht leben wollen, denn Israel ist „ein jüdischer Staat, Refugium für Juden, wenn sie in Not sind." (Z. 45) Nirgendwo sonst auf der Welt können sich Juden vor antisemitischen An- und Übergriffen sicherer fühlen, nirgendwo sonst können sie in der Gewißheit leben, daß eine Massenvernichtung von Juden, wie sie in Nazideutschland geschah, nicht vorkommen werden. 

Vater, den amerikanischen Traum: „Alle ordentlichen Juden fahren nach Amerika [...] Israel hingegen ist kein Land für Juden.“ (Z. 187) Doch im Gegensatz zu Israel sind jüdische Einwanderer in den Vereinigten Staaten nicht willkommen, die Grenzen sind für sie dicht gemacht und der Traum von einem besseren Leben in der neuen Welt ist kaum zu verwirklichen. Trotzdem glaubt die Familie, mit Hilfe der Dienste jüdischer Emigrantenorganisationen und Einwanderungsbüros das Hindernis des Einreisestopps überwinden zu können, was sich aber als Irrtum erweisen sollte. Denn entweder war die Arbeit dieser Büros ineffektiv, wie die des Tolstojfonds in Rom, dessen Aufgabe es gewesen wäre, speziell jüdischen Emigranten aus der Sowjetunion zu einer ständigen Aufenthaltsgenehmigung in den USA zu verhelfen, seine Mitarbeiter aber den Bittstellern immer nur mitteilten, sie würden für sie nichts machen können. Oder es handelte sich um von orthodoxen Juden geführte Organisationen, wie die Vereinigung „Der glückliche Rebbe“ des Rav Pelzer in Wien. Dieser verlangte nicht nur vom Erzähler, er „solle in Amerika eine jüdische Schule besuche, solle [sich] Pejes wachsen lassen und den Talmud studieren“, (Z. 189) als Bedingung dafür, daß er der Familie halft, nach Amerika zu emigrieren. Diese Organisationen arbeiteten überdies mit illegalen Mitteln: Mit Hilfe eines amerikanischen Konsuls in Luxemburg, der gegen ein angemessenes Bestechungsgeld Touristenvisa für auswanderungswillige Juden ausstellte, schleusten sie Migranten aus der Sowjetunion, Rumänien, Bulgarien oder Ungarn ohne gültige Einwanderungspapiere nach Amerika, unterstützen sie dort, indem sie ihnen anfangs eine Wohnung finanzierten und warteten, daß die USVerwaltung die Situation dieser Menschen legalisierte. Als Gegenleistung für die Hilfe erwarten Hilfsorganisationen von den Emigranten, daß sie ein Leben nach den strengen Regeln der Chassiden führen, wie Rav Pelzer es vom Erzähler gefordert hatte. dort aber nicht auf die Hilfe der orthodoxen Juden in Anspruch, sondern läßt sich vorerst in der Russenkolonie „Little Odessa“ in Brooklyn nieder. Später, als das Touristenvisum abgelaufen ist, muß sie in den Untergrund und findet bei Bekannten in der Nähe von Boston Aufnahme, bis sie von den amerikanischen Behörden aufgegriffen und nach Österreich abgeschoben werden.

Der Erzähler fühlt sich in Brooklyn sehr schnell wohl, nicht zuletzt auch deshalb, weil die Familie - gegen den Willen der Mutter - die Hilfe der Chassiden nicht in Anspruch genommen hat und den Befürchtungen des Erzählers, er müsse sich nun Pejes wachsen lassen und er werde beschnitten (Z. 189), somit jede Grundlage entzogen wurde. In „Little Odessa“ kann er Russisch sprechen, russische Bücher und Zeitschriften lesen und ist von ähnlichen Menschen wie in den Transitlagern und in Israel umgeben: „Nun bin ich also in Brooklyn, habe aber mit ,Kaftanjuden' und anderen einheimischen Juden wie Nichtjuden - nur wenig zu tun gehabt. In Little Odessa treffe ich stattdessen immer wieder auf [den] Menschentyp [des] entwurzelten Sowjetmenschen." (Z. 190). Der Erzähler stößt nicht nur auf ähnliche Menschen wie auf seinen Stationen nach New York, auch die Erfahrung, in einer Art Ghetto zu leben, ist ihm von der ersten Ankunft in Wien schon bekannt. „Little Odessa“ ist nicht von Mauern begrenzt, doch die unsichtbaren Schranken sind deswegen nicht weniger reell. Die 7th Avenue ist eine solche Grenzlinie, „die man nicht überqueren durfte“ (Z. 203), und nur völlig ahnungslose Neuankömmlinge setzten sich der Gefahr aus, überfallen und verprügelt $\mathrm{zu}$ werden und wagten sich in die benachbarten Viertel der Schwarzen und 
Puertoricaner. Trotz dieser Widrigkeiten lassen sich die illegalen Einwanderer die Idee vom Glück in Amerika nicht nehmen, auch wenn sie zu ihrer Verteidigung „rassistisch, depressiv oder einfach meschugge werden.“ (Z 205) Der Rassismus gegen die „negroe“ (Z. 203) ist aber nichts anderes als ein Ausdruck der Ohnmacht, mit der sie den Aggressionen der Farbigen gegenüberstehen.

Ganz konkrete Grenzen erfährt der Erzähler in seiner amerikanischen Existenz dadurch, daß er keine Sozialversicherungsnummer hat, und deshalb am öffentlichen Leben nicht teilhaben kann, bzw. ganz konkret von der Benutzung der Leihbibliothek ausgeschlossen ist. Der Versuch, sich mit einer falschen Nummer Zutritt $\mathrm{zu}$ verschaffen, ist nur kurzzeitig von Erfolg gekrönt, denn sehr bald wird aufgedeckt, daß er die Nummer einer 1899 geborenen Mrs. Freeman angegeben hat. In diesem Moment ist seine Enttarnung für den Erzähler aber nicht mehr von Belang, da er bereits kurze Zeit später gemeinsam mit seinen Eltern in Polizeigewahrsam genommen und nach Österreich abgeschoben werden wird

Im Laufe der Wanderung durch diverse Staaten und Kontinente wird Wien der Fixpunkt, zu dem die Familie, gegen ihren Willen, immer wieder zurückkehrt, nicht zuletzt auch deswegen, weil sie im Besitz österreichischer Aufenthaltspapiere ist. Der erste Grund, weswegen Österreich immer nur eine Durchzugsstation der jüdischen Migranten bleibt, ist die österreichische NS-Vergangenheit. Alle Österreicher ab einem bestimmten Alter könnten an der Vernichtung der Juden beteiligt gewesen sein, und es war nie ausgeschlossen, daß man auf der selben Etage wie der Mörder seiner eigenen Familie wohnte. So möchte auch der Vater des Erzählers unter keinen Umständen „in Österreich [...] leben, weil Hitler und alle wichtigen Nazis dort geboren sind'“ (Z. 82). Dementsprechend groß ist daher die Aufregung in der Familie, als der Erzähler, unwissend, worum es sich bei diesem Gegenstand handelt, eines Tages ein silbernes Zigarettenetui nach Hause bringt, auf dessen Vorderseite die Umrisse von "Großdeutschland“ eingraviert. Wie sich herausstellt, ist es ein Geschenk der Nachbarin Ernestine Berger, bei der der Erzähler seine Nachmittage verbringt, während die Eltern arbeiten. Die schlesische Aussiedlerin, die durch den Krieg fast alle ihre Verwandten verloren hat und ihr Land verlassen mußte, hütet eine Reihe von Erinnerungsstücke aus der NS-Zeit wie heilige Reliquien und erzählt dem Jungen, wie der Führer "die Polacken in die Schranken gewiesen [...] und Europa vor dem Bolschewismus gerettet“ (Z. 52) habe. Aus ihrer Sicht teilt sie als "Opfer des Bolschewismus" das gleiche Schicksal wie die Familie des Erzählers.

Im Gegensatz zum Vater, der von größtem Entsetzen erfaßt wird und sich in seinem Vorurteil über Österreich bestätigt sieht, als er erfährt, daß sein Sohn seine Nachmittage in der Obhut einer Nationalsozialistin verbringt, legt die Mutter eine viel pragmatischere Haltung an den Tag. Sie sieht in der Nachbarin in erster Linie eine kostenlose Kinderbetreuerin, und dabei ist es für sie ganz unwesentlich, ob diese mit den Nazis sympathisiert oder nicht. Kurz nach dem Vorfall erkrankt Ernestine Berger schwer und stirbt bald darauf. Ihr Tod löst das konkrete Problem des Vaters und steht emblematisch dafür, daß Österreich nur durch ihr langsames Aussterben von seinen Nazisympathisanten befreit werden kann. 
20 Wien charakterisiert sich überdies auch durch seine Fremdenfeindlichkeit, die in den verschiedensten Lebensbereichen zu spüren ist, wobei sich die Wiener bei den Ausländern entschuldigen, daß sie xenophob sind, aber leider nicht anders handeln können, wie das an der Haltung eines Immobilienmaklers bei einer Wohnungsbesichtigung klar wird:

„Sie schauen zwar wie eine ordentliche Ausländerin aus“, hatte ein Makler, ein immerfort lächelnde uns äußerst wortreicher Mensch, Mutter einmal erklärt. „Sie sind keine Türkin und auch keine Jugoslawin. Aber es tut mit trotzdem leid. Der Hausherr hat extra betont, daß er keine Ausländer haben will.“ (Z. 56)

Ausländer werden also in zwei Kategorien eingeteilt, in die nicht ordentlichen, zu denen Türken und Jugoslawen gehören und denen die Wiener mit extremer Ablehnung gegenüberstehen, und in die ordentlichen, womit alle anderen gemeint sind, doch auch die Zugehörigkeit zu den ordentlichen Ausländern ändert nichts an der Tatsache, daß es sich um Ausländer handelt und also abgelehnt werden. Diese Xenophobie begegnet dem Erzähler auch in der Schule, wo er auf recht zweifelhafte Weise belobigt wurde, er sei nämlich „besser als diese Türken“ (z. 58), und den Eltern am Arbeitsplatz. Sie sind nicht nur gezwungen, Arbeit anzunehmen, die weit unter ihrer Qualifikation liegt und nicht annähernd ihrer Tätigkeit in der Sowjetunion entspricht, wo der Vater als Jurist und die Mutter als Physikerin und Mathematikerin gearbeitet hat. Jetzt verdienen sie ihr Leben als Magazinarbeiter in der Universitätsbibliothek und als Putzfrau in einer Versicherungsgesellschaft. Und obwohl die Mutter des Erzählers ein besseres Deutsch spricht als ihre Wiener Chefin, die keinen hochdeutschen Satz fehlerfrei sagen konnte, verständigt sich diese mit ihr nur in einem rudimentären Deutsch, weil ein slawischer Akzent für die Wienerin gleichbedeutend ist intellektueller Inferiorität und Faulheit, die Überdies ist die Integration in die Wiener jüdische Gemeinde für die Familie sehr schwierig, denn von der einstmals bedeutenden jüdischen Bevölkerung hat fast niemand den Zweiten Weltkrieg überlebt; eine engere Bindung zu den orthodoxen Juden kommt für die laizistisch eingestellte Familie nicht in Frage, zumal ihnen diese genauso fremd sind wie den Nichtjuden. Von den Russen als Juden diskriminiert, von der österreichischen Bevölkerung als Ausländer abgelehnt, ohne wirkliche Beziehungen zum Judentum bleiben sie in einem Niemandsland zwischen den Kulturen. Die Wiener Freundin Rita faßt es so zusammen: „Es ist schon tragisch [...], Russen durftet ihr nicht sein, richtige Juden seid ihr keine mehr, Gojim aber auch nicht." (Z. 280) Der Erzähler zeichnet sich in der Folge durch außerordentliche schulische Leistungen aus, aber auch dadurch, daß er „von den christlichen Ritualen, Weihnachten, Ostern [...] mehr Ahnung als von den jüdischen“ (Z. 279) hat und von den jüdischen Freunden aufgefordert wird, doch die Religion seiner Väter zu studieren.

Der Roman endet damit, daß der Erzähler Wien verläßt, um zu seiner Freundin in Salzburg zu ziehen. War der Beginn eine endgültige Abnabelung von seiner alten Heimat, so steht am Ende die endgültige Abnabelung von den Eltern und damit auch das Ende der Odyssee. Eine Art Normalität stellt sich ein, die auch dadurch gekennzeichnet ist, daß das Verlassen der elterlichen Wohnung von einem kleinen Streit mit den Eltern begleitet ist, die, wie die Mehrzahl der Eltern, nur mit großen Vorbehalten akzeptieren, daß ihr Kind sie verläßt. 

noch in der neuen, fremde Kultur, zurechtfindet und für sich einen von Homi K. Bhabha ${ }^{8}$ so genannten „dritten Raum“ erschließt, den er sich aneignet. Der Roman Vertlibs geht viel weiter, er vergleicht nicht nur das Eigene mit dem Fremden, sondern das eine Fremde mit dem anderen Fremden, denn er macht an vielen Stationen in der Fremde halt. Schließlich stellt er fest, daß ihm das Eigene fremd geworden ist und er sich das Fremde angeeignet hat, was vor allem auch in der Wahl der Sprache, in der er den Roman verfaßt hat, zum Ausdruck kommt.

\begin{abstract}
Zwischenstationenist aber vor allem eine moderne Illustration des Mythos des „Ewigen Juden“, dem Joseph Roth in seinem 1927 erschienen Essay Juden auf Wanderschaft auf den Grund zu gehen versuchte. Roth untersucht darin jene Migrantionsbewegung der Ostjuden, die Westen ihr Glück machen wollten, zu Beginn des 20. Jahrhunderts vor allem in Berlin, Wien und Paris, heute in Amerika und in Israel. Doch das erhoffte Glück und der Wohlstand im Westen erwiesen sich meistens nur als Schimäre, so daß sie, wie Ahasver, auch im Westen nicht zur Ruhe kommen. in Salzburg ansässig ist, glaubt er, daß auch diese Stadt möglicherweise nur eine Durchgangsstation ist, denn Seßhaftigkeit gibt es für Menschen wie ihn nicht wirklich: "Jemand, der eine Emigration in einem so prägenden Alter wie ich durchmachen musste, ist wahrscheinlich immer unterwegs. Ich habe manchmal das Gefühl, als Kind weggefahren, aber nie angekommen zu sein. "
Vladimir Vertlib teilt dieses Schicksal, denn obwohl er inzwischen seit einigen Jahren

\section{NOTES}

1. Vladimir Vertlib: Zwischenstationen, Wien, Deuticke Verlag 1999, S. 280. In der Folge zitiere ich:

Z. und Seitenzahl.

2. Die Theodor-Kramer-Gesellschaft beschäftigt sich mit der Erforschung Theodor Kramers (1897-1958) und darüber hinaus mit der Erforschung des österreichischen Exils. Der Lyriker Kramer mußte 1939 Österreich verlassen und verbrachte die Kriegsjahre im englischen Exil, bevor er 1957, kurz vor seinem Tod, wieder nach Österreich zurückkehrte. Insgesamt verfaßte er an die zehntausend Gedichte.

3. Vladimir Vertlib: Schattenbild, in: Walter Hinderer, Claudia Holy, Heinz Lunzer, Ursula Seeber (Hrsg.): Altes Land, neues Land. Verfolgung, Exil, biographisches Schreiben. Texte zum Erich Fried Symposion 1999, Wien, Verlag ZIRKULAR der Dokumentationsstelle für neuere österreichische Literatur, 1999, hier aus: www.literaturhaus.at/veranstaltungen/friedtage/buch/vertlib.html

4. Am 28. September 1973 entführen palästinensische Terroristen am Bahnhof Marchegg drei jüdische Emigranten aus der Sowjetunion und einen österreichischen Zollbeamten und ließen diese am nächsten Tag wieder frei, nachdem ihnen freier Abzug und die Schließung des Transitlagers Schönau zugesichert wurde. Nach der Schließung war Österreich aber weiterhin ein wichtiges Transitland für die jüdisch-sowjetischen Emigranten.

5. Tanja Busse nennt eine Zahl von 200000 in Deutschland eingewanderten Juden, in: Tanja Busse: „Die Opferrolle wollen wir nicht". Jüdische Einwanderer kommen nach Deutschland, um dem 
russischen Antisemitismus zu entgehen, in: Die Zeit vom 11. 05. 2005, Nr 20. Judith Brandner spricht in der Zeitschrift „Die Gazette“ von 65000 eingewanderten sowjetischen Juden, in: Judith Brandner: Eine untergehende Kultur? In: Die Gazette vom 27. Oktober 2002.

6. Man schätzt, daß etwa 5000-6000 Juden in Österreich geblieben sind.

7. Siehe $3, \mathrm{~S}$

8. Siehe dazu: Homi K. Bhabha: The Location of Culture, London, New York: Routledge, 1994.

9. Heimat im Zwischenbereich. Vladimir Vertlib im Gespräch mit Ellen Presser, in: Illustrierte Neue Welt, April/Mai2006.

\section{RÉSUMÉS}

In seinem Roman Zwischenstationen schildert der österreichische Autor Vladimir Vertlib die Stationen der Odyssee einer sowjetischen jüdischen Migrantenfamilie auf ihrem Weg in den Westen. Enttäuscht von den Lebensbedingungen in Israel, ihrem ursprünglichen Ziel, zieht sie durch halb Europa und versucht in den USA auf illegale Weise einzureisen, bis sie sich schließlich in Wien, trotz widriger Umstände, ständig niederläßt.

Obwohl Vertlib in der Sprache seines neuen Heimatlandes und nicht in seiner Mutterssprache schreibt, ist sein Roman kein typischer Migrantenroman, sondern eher eine aktuelle Version des Mythos vom „Ewigen Juden“.

Dans son roman Zwischenstationen, l'écrivain autrichien Vladimir Vertlib décrit le voyage d'une famille juive issue de l'Union soviétique à travers l'occident. Déçue par les conditions de vie en Israel, leur première destination, cette famille poursuit son voyage à travers l'Europe, et elle essaie même de s'installer clandestinement aux Etats Unis, avant de se fixer définitivement à Vienne, malgré les conditions de vie difficiles qu'elle y trouve.

Bien que Vertlib ait écrit son roman dans la langue de son pays d'adoption et non dans sa langue maternelle, il ne s'agit pas d'une oeuvre typique de la littérature de migration, mais d'une version contemporaine du mythe du « Juif errant ».

\section{INDEX}

Mots-clés : Juif errant

oeuvrecitee Zwischenstationen

\section{AUTEURS}

\section{ALFRED STRASSER}

Université Charles de Gaulle - Lille 3 